\title{
Recent and Future Studies of Circumstellar Matter - A Snapshot
}

\author{
M. Jura \\ Department of Physics and Astronomy, University of California, \\ Los Angeles CA 90095-1562, USA
}

\begin{abstract}
At this meeting, powerful new images and spectroscopy of AGB stars were presented. Theoretical models have advanced, and we are learning more from studies of the pre-solar grains isolated from meteorites.

We suggest that several mass loss mechanisms may be operating in AGB stars: current images imply both spherical winds and highly flattened outflows. There are good arguments that in some cases, a companion may be critical in driving the final outflow. In the near future, a number of extremely powerful new instruments and techniques will be available that will lead to a much deeper understanding of these systems.
\end{abstract}

\section{Introduction}

One of our basic goals is to understand how stars die. As intermediate mass stars evolve, they eject matter into space. We want to know the composition and mass of this ejecta, and its fate as it is returned into the interstellar medium.

At the 1989 conference here in Montpellier on AGB stars (see Mennessier \& Omont 1989), there had been considerable progress in understanding the circumstellar matter around AGB stars. The results from the IRAS satellite had been released, and we therefore had a much more comprehensive understanding of the dust around these stars. Furthermore, the IRAM $30 \mathrm{~m}$ telescope had been in operation for a few years, and molecular line studies of these stars had been very successful. Some of the basic results that were well known in 1989 were: (1) AGB stars are luminous $\left(\sim 10^{4} \mathrm{~L}_{\odot}\right)$ highly evolved mass-losing red giants, descended from intermediate mass main sequence stars. These stars are about to become Planetary Nebulae and eventually will become white dwarfs. (2) The winds are composed mainly of dust and molecules. (3) Mass loss rates seem to be known to within a factor of 2 or 3 for many stars. Typical values range from $10^{-7} \mathrm{M}_{\odot} \mathrm{yr}^{-1}$ to $10^{-5} \mathrm{M}_{\odot} \mathrm{yr}^{-1}$ with a few stars having even higher mass loss rates. (4) Most AGB stars have relatively low mass loss rates, but most mass is ejected by the relatively few stars with the high mass loss rates. (5) Outflow velocities can be measured to perhaps $10 \%$ with typical values between 10 and $25 \mathrm{~km} \mathrm{~s}^{-1}$.

Even though considerable progress had been made at that time, there were (and still remain) a number of unsolved problems. Although we have reasonable estimates of the mass loss rates from individual stars by modeling the infrared 
and radio data, we still do not have very good understanding of how $d M / d t$, varies as a function of the star's luminosity, mass, composition, pulsational properties, rotation, magnetic field, or binarity. All of these factors can produce some effect on the total mass loss rate. Furthermore, we do not have a very good idea on how $d M / d t$ from a particular star varies as a function of direction (either as clumps or in bipolar flows) or as a function of time - producing multiple shells. We cannot start from first physical principles and compute the total amount of mass lost by the star on the AGB - a fundamental parameter for both stellar evolution and the evolution of the Milky Way.

Even though there are basic theoretical uncertainties, observations have provided considerable insight into the outflows, and we now have a much deeper understanding of the circumstellar matter around mass-losing AGB stars. Below, in the two following sections ("Spectroscopic Observations" and "Imaging Observations") I outline some of the advances that struck me during the conference. This discussion is not comprehensive, but it may at least highlight some of the progress that is being made. In the following two sections, I list some inferences that struck me as relevant to the topic. First, in "Multiple Mechanisms", I address the issue of whether we ought to consider more than one mass-loss mechanism. In the next section, "Some speculative questions", I list some topics which might be worth addressing. Finally, in the last section, I list some future instrumental capabilities.

\section{Spectroscopic observations}

One of our goals is to measure the amounts of the different molecules and particles that are ejected by an AGB star. For example, for a carbon star, we still do not have a very precise accounting of all the carbon - how much is lost in $\mathrm{CO}$ and other small molecules, how much is lost in grains of different sizes and shapes, and how much is lost in intermediate size clusters such as PAH's.

At this conference, results from new instruments - notably the spectrometers on ISO - were presented. The detection of $\mathrm{CO}_{2}$ in the outflows from oxygen-rich stars (Justtanont et al. 1998) is striking. Infrared observations are also necessary to measure the amount of $\mathrm{C}_{2} \mathrm{H}_{2}-\mathrm{a}$ major constituent in the outflows from carbon stars, and progress is now being reported for measuring the amount of this molecule in outflows (see Yamamura et al. 1998). While atomic lines have been detected in emission with the ISO data (Barlow 1998; Fong et al. 1998), a related advance is the ability to detect submillimeter wavelength CI emission by using large ground-based telescopes such as the CSO (see the poster by Crosas et al. 1998)

Another major advance during the past few years has been the study of the solid state features in the spectra of the circumstellar dust (Waters \& Molster 1998). Particularly striking is the detection of crystalline silicates in the dust around $\mathrm{AC} \mathrm{Her,} \mathrm{a} \mathrm{result} \mathrm{that} \mathrm{seems} \mathrm{to} \mathrm{be} \mathrm{best} \mathrm{interpreted} \mathrm{by} \mathrm{assuming} \mathrm{a} \mathrm{long-}$ lived orbiting disk of material (Van Winckel et al. 1998). 


\section{Imaging observations}

Enormous progress has been made in mapping circumstellar matter around stars. Some of the highlights presented at this conference include:

1. The "movie" of the SiO masers around TX Cam achieved with the VLBA (Diamond \& Kemball 1998) shows mostly outflow but also dramatic infall of matter during certain pulsational phases.

2. The near-IR interferometric maps of the Red Rectangle (Osterbart et al. 1997, 1998; Mékarnia et al. 1998) beautifully display both the dusty disk and the $\mathrm{X}$ shaped pattern of what may be a bipolar outflow from the disk. Other optical and near IR interferometry shows intense regions of activity on some stars (Tuthill, Monnier \& Danchi 1998).

3. Radio interferometry at the Plateau de Bure (Lucas \& Guélin 1998) shows molecular structure - including striking rings of molecules around IRC +10216 as expected in the photochemical models (Glassgold 1996).

4. Optical images of the reflection of the diffuse galactic light by the dust around IRC +10216 show multiple shells and extended spherical symmetry (de Laverny, Mauron \& Lopez 1998). The extended emission line scattering around evolved stars with detached shells is also noteworthy (Delgado et al. 1998).

5. The HST optical image of the Egg Nebula (Sahai et al. 1998) shows both more-or-less spherical shells and a very flat disk. What the image shows, but was not clear from previous ground-based studies, is the appearance of two very distinct circumstellar regions. This suggests that two mechanisms may be at work in the mass loss from this object.

6. Ground-based images taken at mid-IR wavelengths often show axially symmetry in Proto Planetary Nebulae (Meixner et al. 1997, 1998).

\section{Multiple mass-loss mechanisms?}

If only for simplicity, there is a preference to think that each star loses mass because of a single mechanism. However, reality is not always this easy. Models demonstrating the coupling between radiation pressure on dust and pulsations are making great progress (Höfner 1998; Fleischer, Winters \& Sedlmayr 1998). These models predict essentially spherically symmetric mass loss and can explain most of the observations of the extended circumstellar envelope around IRC +10216 . In contrast to what is expected from these models, it is clear that some outflows are very axi-symmetric but non-spherical. In the case of the Egg Nebula, the image obtained with the HST points to a very marked contrast between the spherical and flattened circumstellar dust distributions, suggesting two different kinds of outflows.

Clearly, there must be at least some instances where a binary companion plays a key role in the evolution of the mass-losing star. Binaries such as the Red Rectangle (Van Winckel, Waelkens \& Waters 1995; Waelkens et al. 1996) 
and AC Her (Van Winckel et al. 1998) have periods near 1-3 years, and in such evolved systems, the companion must have played an important role in driving the mass loss (see, for example, Taam 1996). While there have been extensive discussions of the role of binaries in outflows (Soker 1998; Jorissen 1998), we still do not have a full understanding of when and how this occurs.

In following the mass loss process, we should not only consider the energetics but also follow the angular momentum history as well. The frequency of axi-symmetric outflows suggests that there are often instances when angular momentum transfer plays a key role in the mass loss.

\section{Some speculative questions}

While we study the circumstellar envelopes around AGB stars, there are certain questions that come to mind. By posing and then answering these questions, we may learn more about these systems. I would include the following:

1. When and where do the gas and dust separate from each other in some circumstellar regions? Abundance analysis of several post-AGB stars shows a remarkable pattern: volatile elements (carbon, nitrogen, oxygen, zinc) have approximately solar abundances while refractory elements (such as iron) are depleted by as much as a factor of $10^{3}$ (Waters, Trams \& Waelkens 1992). The interpretation of these data is that the star has accreted gas but not dust. If so, then the gas and dust have separated.

2. Can planets form around post-AGB stars? Planets have been found around the pulsar PSR $1257+12$ (Wolsczan \& Frail 1992). There appear to be long-lived disks around some post-AGB stars as well, such as the Red Rectangle and AC Her (Jura, Balm \& Kahane 1995; Van Winckel et al. 1998). The mass of dust in these disks is $10^{30} \mathrm{~g}$ - sufficient for planet formation. In the Red Rectangle, there appears to be a large structure in the dust cloud (Jura \& Turner 1998). Can planets form in these objects?

3. Can we account for the powerful X-ray sources associated with the accretion from the outflows from AGB red giants? Accretion of matter onto a compact source such as a white dwarf, neutron star or black hole results in the copious emission of $\mathrm{X}$-rays. There are large numbers of mass-losing red giants in the Milky Way. However, there are very few such red giants such as GX 1+4 (Chakrabarty, van Kerkwijk \& Larkin 1998) associated with X-ray sources more powerful than $10 \mathrm{~L}_{\odot}$. Since so many stars are binaries, there should be large numbers of binary red giants - some of which should have X-ray companions (see, for example, Hunsch et al. 1998). While some symbiotic stars do display X-ray emission, the total X-ray luminosity often is less than $1 \mathrm{~L}_{\odot}$ (Jorissen et al. 1996; Muerset, Wolff \& Jordan 1997). Also, it seems that a few of the "luminous supersoft X-ray Sources" include this class of object - white dwarfs powered by accretion of the wind from an AGB companion (Kahabka \& van den Heuvel 1997). Further study of this class of wide binary where accretion from a red giant wind may occur seems appropriate. 
4. Where are the large (diameters up to $10 \mu \mathrm{m}$ ) inclusions in meteorites formed? As discussed by Anders \& Zinner (1993) and Ott (1993), there is now compelling evidence that some inclusions in meteorites are grains that were formed in the outflows from AGB stars and then survived both their passage through the interstellar medium and the pre-solar nebula. Some of these particles are nearly as large as $10 \mu \mathrm{m}$ in diameter and the "average" size is well over $1 \mu \mathrm{m}$ in diameter (Bernatowicz et al. 1996). Typically, the grains ejected by mass-losing carbon stars such as IRC +10216 have diameters near $0.1 \mu \mathrm{m}$ (Jura 1994). Although there might be a natural selection effect to find larger particles because they are more durable, it is surprising that such large particles are created in standard winds. Could it be that such particles are formed in long-lived disks around post-AGB binaries? (Jura, Turner \& Balm 1997)?

5. Are there non-standard mass-losing AGB systems? One of the surprising results from the IRAS data was the discovery that a few carbon-rich stars, such as BM Gem, possess oxygen-rich dust (Little-Marenin 1986; Willems $\&$ de Jong 1986). Are there other unexpected objects?

6. Can we identify the carriers of the diffuse interstellar bands in the AGB outflows? The carriers of the Diffuse Interstellar Bands is an unsolved mystery (Herbig 1995). The nebulosity in the Red Rectangle has a remarkable and nearly unique set of emission bands longward of $5800 \AA$ (Schmidt, Cohen \& Margon 1980). Similar emission has been found to date only in one other star, the R CrB star V854 Cen at minimum light (Kameswara-Rao \& Lambert 1993). It seems possible that the carrier of these bands is related to at least some of the Diffuse Interstellar Bands (Sarre, Miles \& Scarrott 1995). Can we learn more from extending this kind of work? A related topic is the question of the origin of the Extended Red Emission which has been found throughout the Milky Way (Gordon, Witt \& Friedmann 1998; Szomoru \& Guhathakurta 1998), and which may be related to the large continuum bump seen longward of $5800 \AA$ in the spectrum of the Red Rectangle.

\section{The future?}

Progress in instrumentation almost inevitably will lead to advances in our astrophysical understanding. Consider some of the following which will improve our understanding of AGB stars and their winds:

1. New telescopes such as SIRTF, SOFIA, the sub-mm array and the millimeter array and various infrared and optical interferometers will provide extremely powerful tools for studying these stars.

2. New instruments such as SCUBA and the mid-IR imagers (such as MIRLIN and OSCIR) greatly enhance the capability of existing telescopes.

3. The 2MASS and DENIS surveys, as well as further exploitation of the HIPPARCOS data, and then the cross correlation of the surveys with 
each other, should allow a much more comprehensive view of the AGB phenomena.

4. Progress in computers will allow for much more sophisticated modeling.

5. Studies of the meteoritic inclusions are progressing rapidly, and they provide a unique set of insights into the physics of these stars.

In summary, we have made such progress during the past ten years, and I am very optimistic that the next ten years will be a very exciting time to study these stars.

Acknowledgments. This work was partly supported by NASA. I thank the organizers of the conference for designing such an interesting and timely program.

\section{References}

Anders E., Zinner E., 1993, Meteoritics 28, 490

Barlow M.J., 1998, IAU Symposium No. 191, this volume

Bernatowicz T.J., Cowsik R., Gibbons P.C., Lodders K., Fegley B., Amari S., Lewis R.S., 1996, ApJ 472, 760

Chakrabarty D., Van Kerkwijk M.H., Larkin J.E., 1998, ApJ 497, L39

Crosas M., Young K., Ivezić Ž., Knapp G.R., 1998, poster contribution, IAU Symposium No. 191

de Laverny P., Mauron N., Lopez B., 1998, poster contribution, IAU Symposium No. 191

Delgado D.G., Olofsson H., Schwarz H., Eriksson K., Gustafsson B., 1998, poster contribution, IAU Symposium No. 191

Diamond P.J., Kemball A.J., 1998, IAU Symposium No. 191, this volume

Fleischer A.J., Winters J.M., Sedlmayr E., 1998, IAU Symposium No. 191, this volume

Fong D., Meixner M., Sutton E., et al., 1998, poster contribution, IAU Symposium No. 191

Gordon K.D., Witt A.N., Friedmann B.C., 1998, ApJ 498, 522

Glassgold A.E., 1996, ARA\&A 34, 241

Herbig G.H., 1995, ARA\&A 33, 19

Höfner S., 1998, IAU Symposium No. 191, this volume

Hunsch M., Schmitt J.H.M.M., Schröder K.-P., Zickgraf F.-J., 1998, A\&A 330, 225

Jorissen A., 1998, IAU Symposium No. 191, this volume

Jorissen A., Schmitt J.H.M.M., Carquillat J.M., Ginestet N., Bickert K.F., 1996, A\&A 306, 467

Jura M., 1994, ApJ 434, 713

Jura M., Balm S.P., Kahane C.,1995, ApJ 453, 721

Jura M., Turner J.L., 1998, Nature 395, 144 
Jura M., Turner J.L., Balm S.P.,1997, ApJ 474, 741

Justtanont K., Feuchtgruber H., de Jong T., Cami J., Waters L.B.F.M., Yamamura I., Onaka T., 1998, A\&A 330, L17

Kahabka P., van den Heuvel E.P.J., 1997, ARA\&A 35, 69

Kameswara-Rao N., Lambert D.L., 1993, MNRAS 263, 27P

Little-Marenin I.R., 1986, ApJ 307, L15

Lucas R., Guélin M., 1998, IAU Symposium No. 191, this volume

Meixner M., Skinner C.J., Graham J.R., Keto E., Jernigan J.G., Arens J.F., 1997, ApJ 482, 897

Meixner M., Ueta T., Dayal A., Hora J., Fazio G., Hrivnak B., Skinner C.J., Hoffmann W.F., Deutsch L.K., 1998, ApJS, in press

Mékarnia D., Rouan D., Tessier E., Dougados C., Lefèvre J., 1998, A\&A 336, 648

Mennessier M.O., Omont A. (eds.), 1989, From Miras to Planetary Nebulae: Which Path for Stellar Evolution?, Editions Frontières

Muerset U., Wolff B., Jordan S., 1997, A\&A 319, 201

Osterbart R., Langer N., Weigelt G., 1997, A\&A 325, 669

Osterbart R., Balega Y.Y., Langer N., Menshchikov A.B., Weigelt G., 1998, poster contribution, IAU Symposium No. 191

Ott U., 1993, Nature 364, 25

Sahai R., et al., 1998, ApJ 493, 301

Sarre P., Miles J.R., Scarrott S.M., 1995, Science 269, 674

Schmidt G.D., Cohen M., Margon B., 1980, ApJ 239, L133

Soker N., 1998, ApJ 496, 827

Szomoru A., Guhathakurta P., 1998, ApJ 494, L93

Taam R.E., 1996, in Compact Stars in Binaries, ed. J. van Padadijs, E.P.J. van den Heuvel and E. Kuulkers, IAU Symposium No. 165, Kluwer, Dordrecht, p. 3

Tuthill P.G., Monnier J.D., Danchi W.C., 1998, IAU Symposium No. 191, this volume

Van Winckel H., Waelkens C., Waters L.B.F.M., 1995, A\&A 293, L25

Van Winckel H., Waelkens C., Waters L.B.F.M., Molster F.J., Udry S., Bakker E.J., 1998, A\&A 336, L17

Waelkens C., Van Winckel H., Waters L.B.F.M., Bakker E.J., 1996, A\&A 314, L17

Waters L.B.F.M., Molster F.J., 1998, IAU Symposium No. 191, this volume

Waters L.B.F.M., Trams N.R., Waelkens C., 1992, A\&A 262, L37

Wolsczan A., Frail D.A., 1992, Nature 355, 145

Willems F.J., de Jong T., 1986, ApJ 309, L39

Yamamura I., de Jong T., Waters L.B.F.M., Cami J., Justtanont K., 1998, IAU Symposium No. 191, this volume 


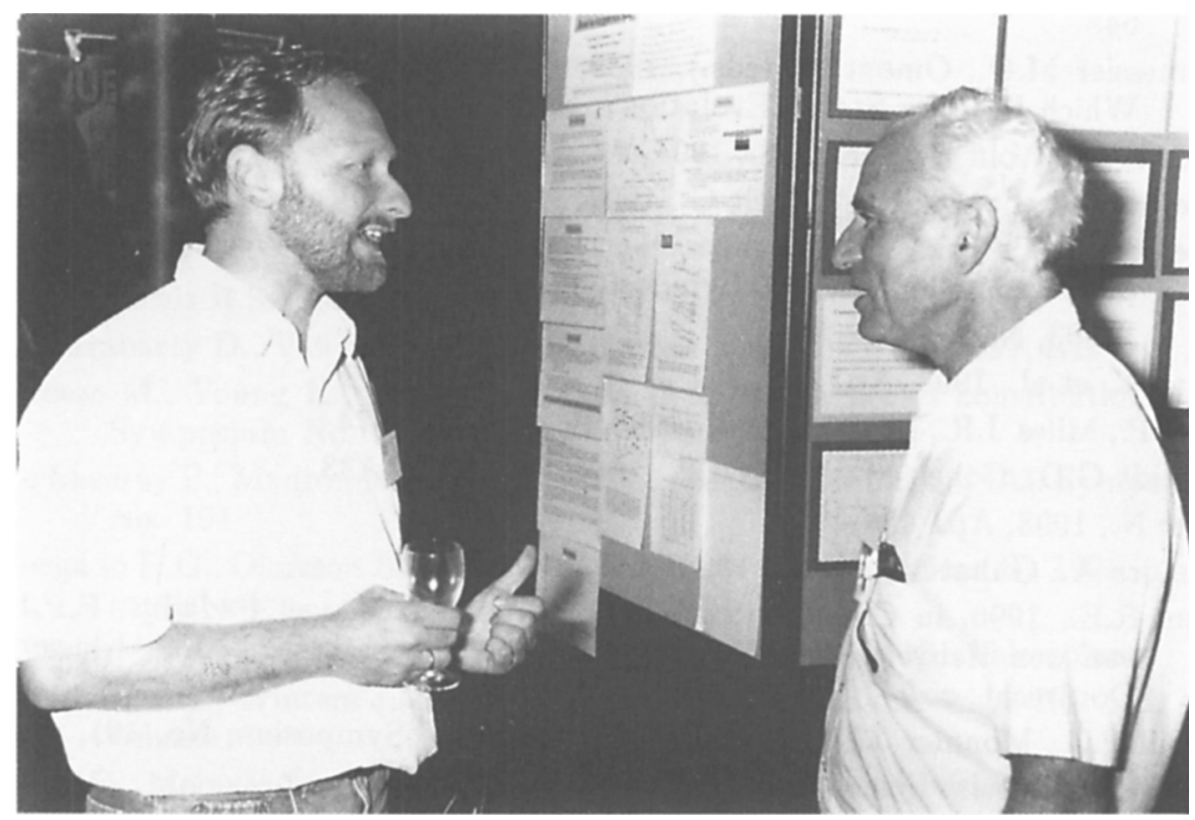

Franz Kerschbaum and Nathan Netzer, poster-sessioning 